\title{
Bufalin Alters Gene Expressions Associated DNA Damage, Cell Cycle, and Apoptosis in Human Lung Cancer NCI-H460 Cells in Vitro
}

Shin-Hwar Wu ${ }^{1,2}$, Yung-Ting Hsiao ${ }^{3}$, Jaw-Chyum Chen ${ }^{4}$, Ju-Hwa Lin ${ }^{3}$, Shu-Chun Hsu ${ }^{3}$, Te-Chun Hsia ${ }^{5,6}$, Su-Tso Yang ${ }^{7}$, Wu-Huei Hsu ${ }^{6,8, *}$ and Jing-Gung Chung ${ }^{3,9, *}$

1 Institute of Clinical Medical Science, China Medical University, Taichung 40402, Taiwan

2 Division of Critical Care Medicine, Department of Medicine, Changhua Christian Hospital 50006, Taiwan

3 Department of Biological Science and Technology, China Medical University, Taichung 40402, Taiwan

4 Department of Medicinal Botany and Health Applications, Da-Yeh University, Changhua 51591, Taiwan

5 Gradualted Institute of Chinese Medical Science, China Medical University, Taichung 40402, Taiwan

6 Department of Internal Medicine, China Medical University Hospital, Taichung 40447, Taiwan

7 Department of Radiology, China Medical University Hospital, Taichung 40447, Taiwan

8 Department of Internal Medicine, China Medical University, Taichung 40402, Taiwan

9 Department of Biotechnology, Asia University, Taichung 41354, Taiwan

* Authors to whom correspondence should be addressed; E-Mails: hsuwh@mail.cmuh.org.tw (W.-H.H.); jgchung@mail.cmu.edu.tw (J.-G.C.); Tel.: +886-42205-3366 (ext. 3483) (W.-H.H.); +886-4-2205-3366 (ext. 2161) (J.-G.C.); Fax: +886-42203-8883 (W.-H.H.); +886-4-2205-3764 (J.-G.C.).

Received: 12 March 2014; in revised form: 2 May 2014 / Accepted: 8 May 2014 /

Published: 13 May 2014

\begin{abstract}
Lung cancer is the leading cause of cancer related death and there is no effective treatment to date. Bufalin has been shown effective in inducing apoptosis and DNA damage in lung cancer cells. However, the genetic mechanisms underlying these actions have not been elucidated yet. Cultured NCI-H460 cells were treated with or without $2 \mu \mathrm{M}$ of bufalin for $24 \mathrm{~h}$. The total RNA was extracted from each treatment for cDNA synthesis and labeling, microarray hybridization, and then followed by flour-labeled cDNA hybridized on chip. The localized concentrations of fluorescent molecules were detected and quantitated and analyzed by Expression Console software (Affymetrix) with default RMA parameters. The key genes involved and their possible interaction pathways were mapped by GeneGo software. About 165 apoptosis-related genes were affected. CASP9
\end{abstract}


was up-regulated by 5.51 fold and THAP1 by 2.75 -fold while CCAR1 was down-regulated by 2.24 fold. 107 genes related to DNA damage/repair were affected. MDC1 was down-regulated by 2.22 -fold, DDIT4 by 2.52 fold while GADD45B up-regulated by 3.72 fold. 201 genes related to cell cycles were affected. CCPG1 was down-regulated by 2.11 fold and CDCA7L by 2.71 fold. Many genes about apoptosis, cell cycle regulation and DNA repair are changed significantly following bufalin treatment in NCI-H460 cells. These changes provide an in depth understanding of cytotoxic mechanism of bufalin in genetic level and also offer many potentially useful biomarkers for diagnosis and treatment of lung cancer in future.

Keywords: bufalin; cDNA microarray; DNA damage; cell cycle; apoptosis; NCI-H460 cells

\section{Introduction}

Non-small cell lung carcinoma is one of leading causes of cancer-related death in the United States and throughout the world [1]. It causes more than one million deaths every year [2]. Most non-small cell lung cancer patients are diagnosed at late stages (stage IIIb or IV) and are inoperable. Standard platinum-based chemotherapies provide marginal improvement in survival at the expense of substantial toxicities [3]. Even with the addition of target-therapy, the median survival of metastatic non-small cell lung cancer patients is about one year [4]. Because of the unsatisfactory results of standard chemotherapy, many advocate finding new drugs.

Natural products were the main source of health care in ancient times. In modern medicine, they are still major sources of new drug development. Chan $\mathrm{Su}$ is derived from the serous fluid of the posterior auricular glands of toad. Its anti-tumor effects have been reported in many Chinese treatises. Bufalin is thought to be the key component of Chan Su. Modern researches also confirm bufalin-induced cytotoxic effects on many human cancer cells [5-9]. The mechanisms of action of bufalin on cancer cell have been shown to include apoptosis-induction [10], interruption of damaged DNA repair [11], halting uncontrolled cell cycle [12] and inhibition of cancer cell migration/metastasis.

Genetic mutations play a pivotal role in oncogenesis. Virtually all human cancers have mutations interfering cell cycle checkpoints and leading to uncontrolled cell growth. Some oncogenic mutations involving inactivating apoptosis or disrupting DNA repair mechanism. These vulnerable genes are thus targets of detection or treatment for various cancers.

There is little research exploring the mechanisms of bufalin's anti-cancer effects at a genetic level. By Northern blot or RT-PCR, these studies focused on less than five genes at one time $[6,13,14]$. There have been no studies using cDNA microarrays for extensive genetic surveys for this purpose till now. In this study, we used cDNA microarrays to investigate the genetic change of NCI-H460 lung cancer cells following bufalin treatment in vitro. We put special emphases on genes related to cell cycle regulation, apoptosis induction and DNA repair. Through this study, we are closer to understand the mechanisms underlying bufalin's anti-cancer effects at the genetic level. We hope this knowledge will provide a basis for future studies on anti-cancer drug development or modification. 


\section{Results and Discussion}

\subsection{The Up-Regulated and Down-Regulated Gene Expression in H460 Exposed to Bufalin}

H460 cells were treated with or without $2 \mu \mathrm{M}$ of bufalin in 12 well-plates for $24 \mathrm{~h}$, then cells were harvested and total RNA were extracted and measurement of concentration and then cDNA microarray analysis of gene expression was performed. The calculated gene expressions from microarrays are shown in Table 1. Table 1 indicates that six genes were over 20 -fold and 21 were over 10 -fold up-regulated. Eleven genes were down-regulated over 10-fold and 42 genes over 6-fold. Table 2 shows the descriptions of genes highly influenced by bufalin treatment. Among those affected genes, 165 are associated with apoptosis, such as CASP9 (caspase 9, apoptosis-related cysteine peptidase) which was up-regulated 5.51-fold. THAP1 (THAP domain containing, apoptosis associated protein 1) was up-regulated by 2.75 -fold. CCAR1 (cell division cycle and apoptosis regulator 1) was down-regulated by 2.24-fold. One hundred and seven affected genes are associated with DNA damage and repair, such as MDC1 (mediator of DNA-damage checkpoint 1), that was down-regulated by 2.22-fold or DDIT4 (DNA-damage-inducible transcript 4) which was suppressed by 2.52-fold, whereas, GADD45B (growth arrest and DNA-damage-inducible, beta) was up-regulated by 3.72-old. As for genes related to cell cycle regulation, 201 were affected. For example, CCPG1 (cell cycle progression 1) was down-regulated by 2.11-fold and CDCA7L (cell division cycle associated 7-like) was inhibited by 2.71-fold.

Table 1. Number of genes by the fold change after bufalin treatment.

\begin{tabular}{cc}
\hline Fold Change & Number of Genes \\
\hline$\geq 20$ & 6 \\
$\geq 10$ and $<20$ & 21 \\
$\geq 4$ and $<10$ & 59 \\
$\geq 3$ and $<5$ & 53 \\
$\geq 2$ and $<3$ & 118 \\
$>-3$ and $\leq-2$ & 488 \\
$>-4$ and $\leq-3$ & 1215 \\
$>-5$ and $\leq-4$ & 348 \\
$>-6$ and $\leq-5$ & 99 \\
$>-10$ and $\leq-6$ & 57 \\
$<-10$ & 42 \\
\hline
\end{tabular}

2.2. GeneGo Analysis Program from Bufalin Treated H460 Cells Demonstrated the Top Alteration in Gene Expression Scored by the Number of Pathway Networks

The results from GeneGo analysis are shown in Figures 1-3. Experimental data were mapped on the processes and shown as red (up-regulation) and blue (down-regulation) circles of different intensities indicating different inhibitions in NCI-H460 cell after bufalin treatment. 
Table 2. Representative genes of NCI-H460 were influenced by bufalin.

\begin{tabular}{|c|c|c|}
\hline Fold change & Gene Symbol & mRNA Description \\
\hline 66.33 & EGR1 & early growth response 1 \\
\hline 26.16 & EGR2 & GTP binding protein overexpressed in skeletal muscle \\
\hline 24.09 & EGR3 & pyruvate dehydrogenase kinase, isozyme 4 \\
\hline 23.48 & EGR4 & NOTCH-regulated ankyrin repeat protein \\
\hline 23.10 & EGR5 & histone cluster $1, \mathrm{H} 4 \mathrm{~d}$ \\
\hline 20.63 & EGR6 & FBJ murine osteosarcoma viral oncogene homolog \\
\hline 5.52 & BTG & BTG family, member 2 \\
\hline 5.51 & CASP9 & caspase 9 , apoptosis-related cysteine peptidase \\
\hline 4.54 & CRY2 & cryptochrome 2 (photolyase-like) \\
\hline 4.36 & AEN & apoptosis enhancing nuclease \\
\hline 3.72 & GADD45B & growth arrest and DNA-damage-inducible, beta \\
\hline 2.75 & THAP1 & $\begin{array}{l}\text { THAP domain containing, apoptosis associated protein } 1 \\
\text { similar to Asparagine synthetase [glutamine-hydrolyzing] }\end{array}$ \\
\hline 2.02 & FLJ43315 & $\begin{array}{l}\text { (Glutamine-dependent asparagine synthetase) (TS11 cell } \\
\text { cycle control protein) }\end{array}$ \\
\hline-2.11 & CCPG1 & cell cycle progression 1 \\
\hline-2.22 & MDC1 & mediator of DNA-damage checkpoint 1 \\
\hline-2.24 & CCAR1 & cell division cycle and apoptosis regulator 1 \\
\hline-2.25 & SMC3 & structural maintenance of chromosomes 3 \\
\hline-2.52 & DDIT4 & DNA-damage-inducible transcript 4 \\
\hline-2.71 & CDCA7L & cell division cycle associated 7-like \\
\hline-3.18 & DCLRE1C & DNA cross-link repair 1C (PSO2 homolog, S. cerevisiae) \\
\hline-3.93 & UIMC1 & ubiquitin interaction motif containing 1 \\
\hline-5.04 & DCLRE1A & DNA cross-link repair 1A (PSO2 homolog, S. cerevisiae) \\
\hline-10.55 & CCND2 & cyclin D2 \\
\hline-10.57 & C5orf33 & chromosome 5 open reading frame 33 \\
\hline-11.08 & GPR65 & G protein-coupled receptor 65 \\
\hline-11.38 & PRICKLE1 & prickle homolog 1 (Drosophila) \\
\hline-11.93 & ORC5L & origin recognition complex, subunit 5-like (yeast) \\
\hline-12.02 & AHNAK2 & AHNAK nucleoprotein 2 \\
\hline-12.24 & ICT1 & immature colon carcinoma transcript 1 \\
\hline-12.61 & KRT19 & keratin 19 \\
\hline-15.54 & OCLN & occludin \\
\hline-27.38 & MIR1977 & microRNA 1977 \\
\hline
\end{tabular}

\subsection{Discussion}

In this study, we found the gene AEN of NCI-H460 cells was up-regulated by 4.36-fold after bufalin treatment. AEN encodes a protein called 'apoptosis-enhancing nuclease', which is usually located in the nucleolus and is translocated to the nucleoplasm upon stimulation by apoptosis signals. Ionizing radiation or DNA-damaging agents such as adriamycin could induce phosphorylation of Ser-15 of apoptosis-enhancing nuclease by p53 and lead to the activation of the latter. Upon activation, apoptosis-enhancing nuclease exerts an exonuclease function and cleaves double-stranded DNA into single-stranded one to augments p53-induced apoptosis $[15,16]$. 
Figure 1. The top scored (by the number of pathways) AN network from GeneGo 02 . Thick cyan lines indicate the fragments of canonical pathways. Red circles represent up regulated genes and down regulated with blue circles represents down-regulated genes. The 'checkerboard' color indicates mixed expressions for the genes between files or between multiple tags for the same gene.

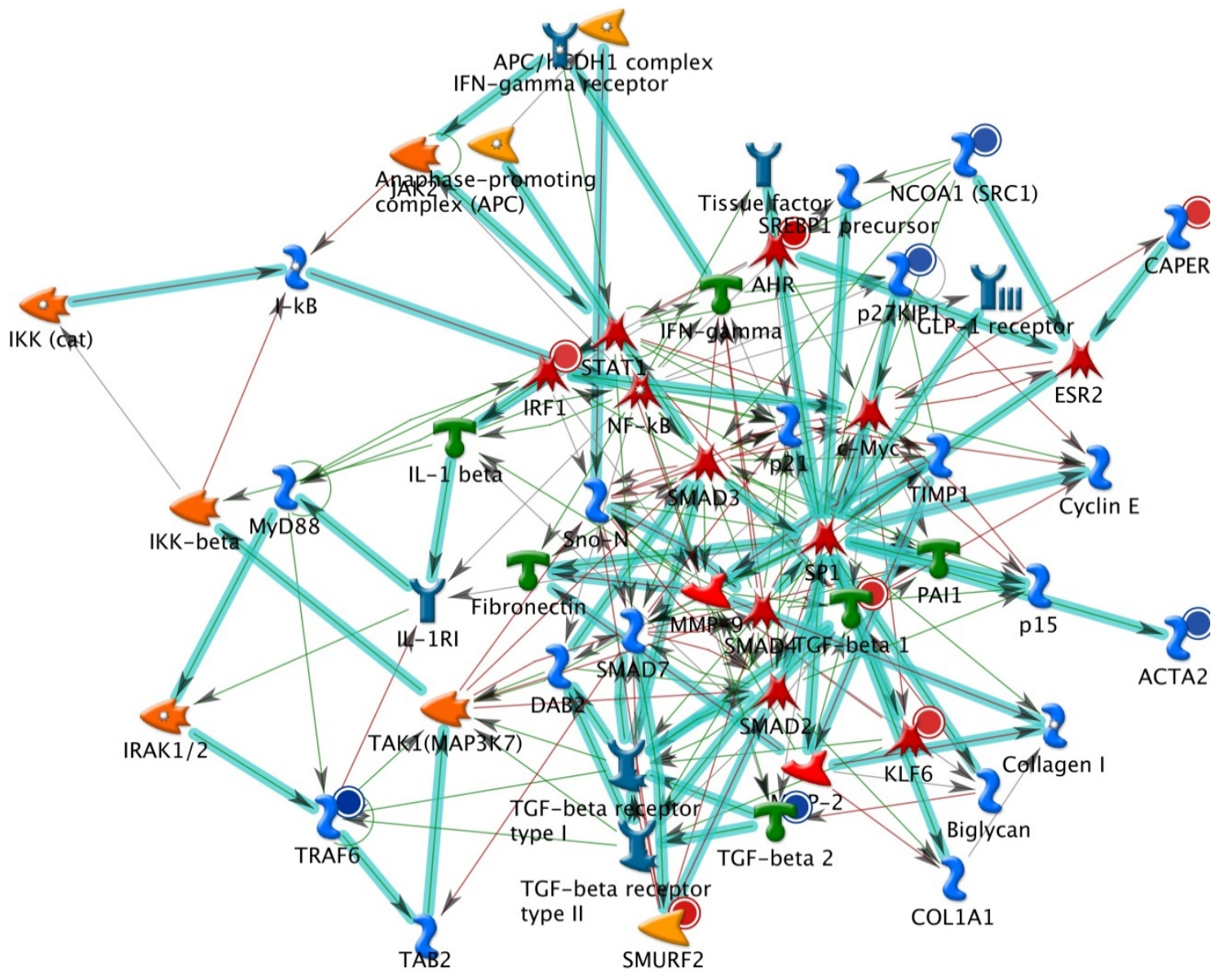

Bufalin treatment also led to a 3.72-fold increase of the gene GADD45B, which is a member of a group of genes usually up-regulated following stressful growth arrest or DNA damage [17]. These genes regulate cell growth and apoptosis by mediating activation of the $\mathrm{p} 38 / \mathrm{JNK}$ pathway via their proteins binding and activation of upstream activator MTK1/MEKK4 kinase. These genes often cooperate with different mechanisms to inhibit cell growth [18]. GADD45B has been found to have potential clinical usefulness as a diagnostic or grading parameter for malignancies [19].

The gene DCLRE1A, also known as SNM1A, was down-regulated by 5.04-fold in NCI-H460 cells treated with bufalin. DCLRE1A encodes the DNA cross-link repair 1A protein (hSNM1A), which is located in the nuclei of cells from various tissues such as brain, heart, kidney, liver, pancreas, placenta and skeletal muscle [20]. This protein belongs to the DNA repair metallo- $\beta$-lactamase family and is involved in DNA inter-strand cross-link repair. It is also required for checkpoint mediated cell cycle arrest in early prophase following mitotic spindle stress [21]. 
Figure 2. The second scored (by the number of pathways) AN network from GeneGo 02. Thick cyan lines indicate the fragments of canonical pathways. Red circles represent up regulated genes and blue circles represent down-regulated genes. The 'checkerboard' color indicates mixed expressions for the genes between files or between multiple tags for the same gene.

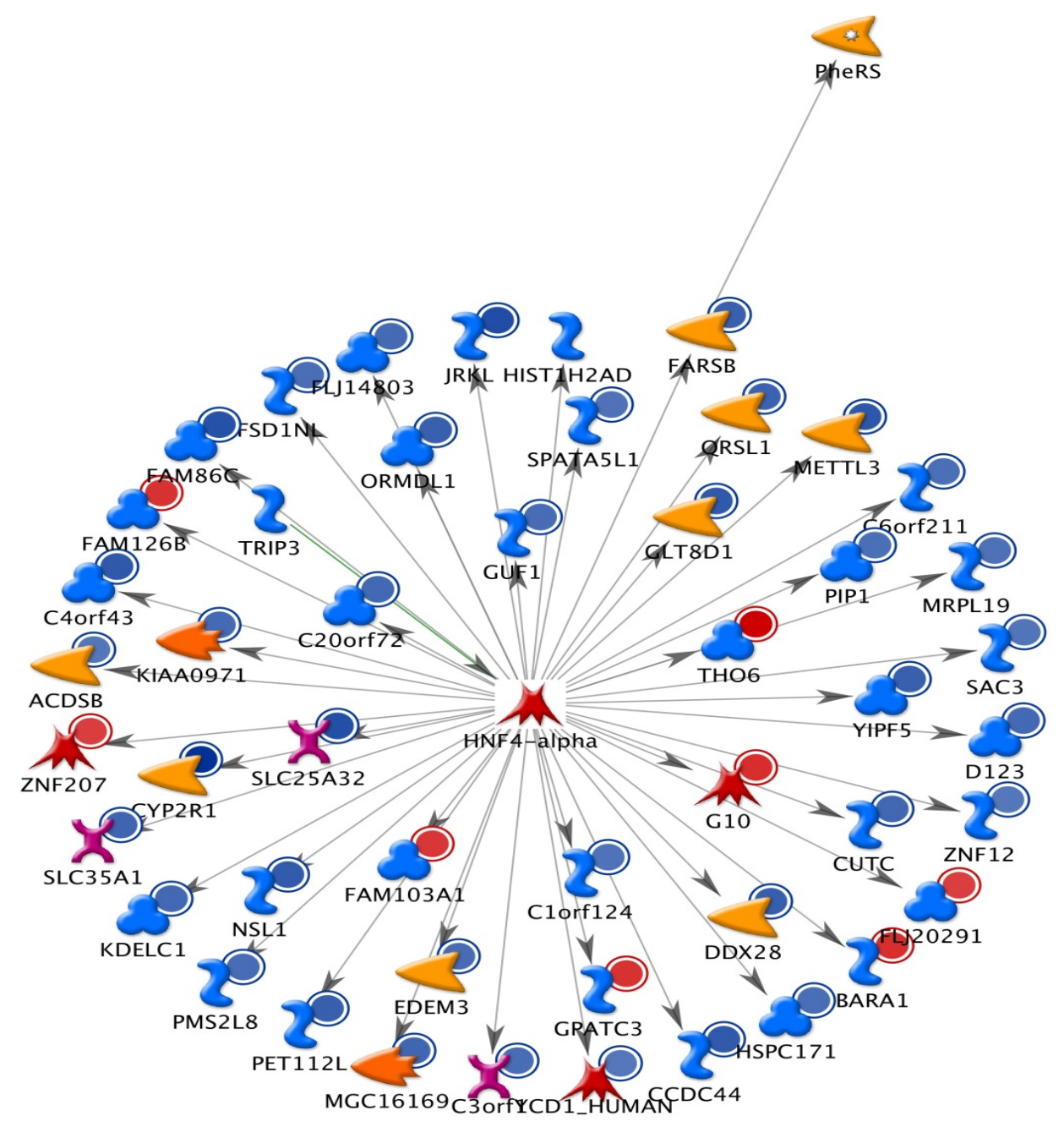

Cyclin D2 is associated with and activates the catalytic activities of cyclin-dependent kinase (CDK) 4 and CDK6 to facilitate cell cycle transition from G1 to $\mathrm{S}$ in mammalian cells. One of the most significant substrates of cyclin D2-CDK complexes is Rb protein, a tumor suppressor protein known for its association with hereditary retinoblastoma. CCND2, the gene encoding cyclin D2, has been found related to several human malignancies including glioblastoma [22], leukemia [23] and ovarian cancers. Over-expression of CCND2 is associated with increased invasiveness in human squamous cell carcinoma in vivo [24]. We found CCND2 gene was down-regulated by 10.55 -fold in NCI-H460 cells treated with bufalin. This substantial suppression may contribute to the cytotoxic and anti-metastasis effects of bufalin on NCI-H460 cells.

Because many genes related to apoptosis, cell cycle regulation and DNA repair are changed following bufalin treatment; we present their complex interactions in Figures 1-3. These figures just represent our understanding based on current knowledge and assumptions. They are not meant to be comprehensive, nor undebatable. More studies are needed to expand our current understanding. 
Figure 3. The third scored (by the number of pathways) AN network from GeneGo 02. Thick cyan lines indicate the fragments of canonical pathways. Red circles represent up regulated genes and blue circles represent down-regulated genes. The 'checkerboard' color indicates mixed expressions for the genes between files or between multiple tags for the same gene.

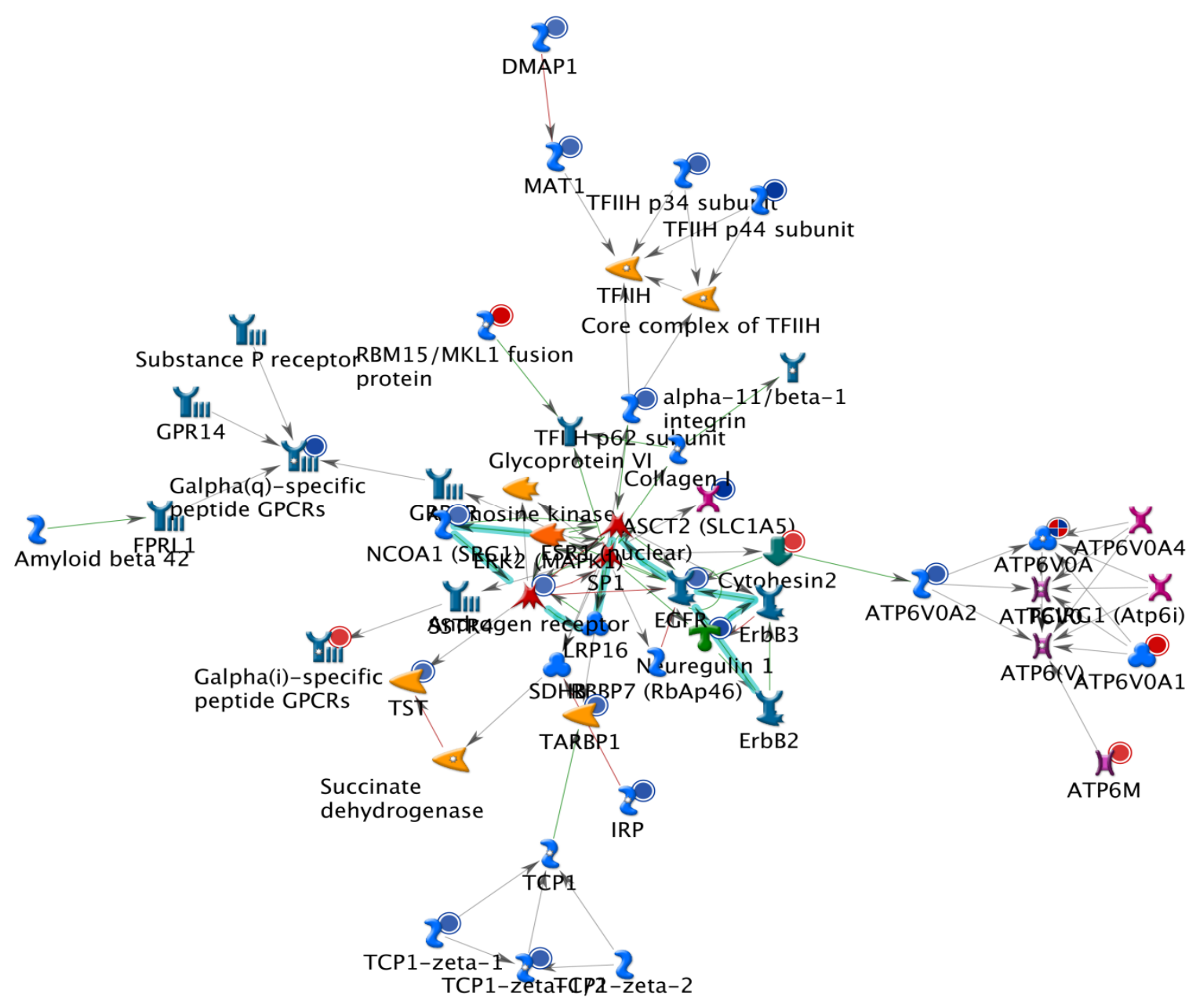

\section{Experimental}

\subsection{Chemicals and Reagents}

Bufalin and dimethyl sulfoxide (DMSO) were purchased from Sigma Chemical Co. (St. Louis, MO, USA). Culture medium RPMI-1640 with additional 10\% fetal bovine serum and 1\% L-glutamine were purchased from Gibco BRL (Grand Island, NY, USA). Bufalin was dissolved in DMSO and stocked at $20^{\circ} \mathrm{C}$.

\subsection{Lung Cancer Cells}

The NCI-H460 human lung cancer cell line was purchased from the Food Industry Research and Development Institute (Hsinchu, Taiwan). It was maintained in a RPMI-1640 medium plus 10\% fetal bovine serum, $1 \%$ L-glutamine, 100 units $/ \mathrm{mL}$ of penicillin $\mathrm{G}$ and $100 \mathrm{microgram} / \mathrm{mL}$ of streptomycin. 
The cells were kept in a $37{ }^{\circ} \mathrm{C}$ incubator under $5 \% \mathrm{CO}_{2}$ and $95 \%$ air. The cells were sub-cultured at $80 \%-90 \%$ confluency [25-27].

\section{3. cDNA Microarray Assay for Genes Expression in H460 Cells after Exposure to Bufalin}

NCI-H460 cells were placed at a density of $5 \times 10^{5}$ cells $/ \mathrm{mL}$ in RPMI 1640 medium for $24 \mathrm{~h}$. Cells were treated without (control) and with $2 \mu \mathrm{M}$ of bufalin for $24 \mathrm{~h}$. The total RNAs were extracted by Qiagen RNeasy Mini Kit (Qiagen, Inc, Valencia, CA, USA) and were used for cDNA synthesis and labeling, microarray hybridization, and then followed by flour-labeled cDNA hybridizing their complements on the chip (Affymetrix GeneChip Human Gene 1.0 ST array, Affymetrix, Santa Clara, CA, USA). Also, the resulting localized concentrations of fluorescent molecules were detected and quantified (Asia BioInnovations Corporation, Taipei, Taiwan). Finally, the resulting data were analyzed by Expression Console software (Affymetrix) with default RMA parameters [28-31]. Genes regulated by bufalin by at least a 2 -fold change were recorded. Data are representative of three separate assays.

\section{Conclusions}

In conclusion, we have demonstrated in this study that many genes connected with apoptosis, cell cycle regulation and DNA repair are changed significantly following bufalin treatment. These changes provide an in-depth understanding of the mechanism(s) of bufalin cytotoxicity in a genetic level and also offer many potentially useful biomarkers for diagnosis and treatment of lung cancer.

\section{Acknowledgments}

This work was supported by grant 100-CCH-IRP-25 from Changhua Christian Hospital (Changhua, Taiwan, ROC).

\section{Author Contributions}

W.-H.H. and J.-G.C. conceived and designed the study. S.-H.W., Y.-T.H., J.-C.C., J.-H.L. and S.-C.H. performed the experiments. J.-G.C. wrote the paper. S.-C.H., T.-C.H. and S.-T.Y. reviewed and edited the manuscript. All authors read and approved the manuscript.

\section{Conflicts of Interest}

The authors declare no conflict of interest.

\section{References}

1. Siegel, R.; Naishadham, D.; Jemal, A. Cancer statistics, 2013. CA Cancer J. Clin. 2013, 63, 11-30.

2. Parkin, D.M.; Bray, F.; Ferlay, J.; Pisani, P. Global cancer statistics, 2002. CA Cancer J. Clin. 2005, 55, 74-108.

3. Chemotherapy in non-small cell lung cancer: A meta-analysis using updated data on individual patients from 52 randomised clinical trials. Non-small Cell Lung Cancer Collaborative Group. BMJ 1995, 311, 899-909. 
4. Sandler, A.; Gray, R.; Perry, M.C.; Brahmer, J.; Schiller, J.H.; Dowlati, A.; Lilenbaum, R.; Johnson, D.H. Paclitaxel-carboplatin alone or with bevacizumab for non-small-cell lung cancer. N. Engl. J. Med. 2006, 355, 2542-2550.

5. Zhu, Z.; Sun, H.; Ma, G.; Wang, Z.; Li, E.; Liu, Y.; Liu, Y. Bufalin induces lung cancer cell apoptosis via the inhibition of PI3K/Akt pathway. Int. J. Mol. Sci. 2012, 13, 2025-2035.

6. Han, K.Q.; Huang, G.; Gu, W.; Su, Y.H.; Huang, X.Q.; Ling, C.Q. Anti-tumor activities and apoptosis-regulated mechanisms of bufalin on the orthotopic transplantation tumor model of human hepatocellular carcinoma in nude mice. World J. Gastroenterol. 2007, 13, 3374-3379.

7. Chen, A.; Yu, J.; Zhang, L.; Sun, Y.; Zhang, Y.; Guo, H.; Zhou, Y.; Mitchelson, K.; Cheng, J. Microarray and biochemical analysis of bufalin-induced apoptosis of HL-60 Cells. Biotechnol. Lett. 2009, 31, 487-494.

8. Chen, H.Y.; Lu, H.F.; Yang, J.S.; Kuo, S.C.; Lo, C.; Yang, M.D.; Chiu, T.H.; Chueh, F.S.; Ho, H.C.; Ko, Y.C.; et al. The novel quinolone CHM-1 induces DNA damage and inhibits DNA repair gene expressions in a human osterogenic sarcoma cell line. Anticancer Res. 2010, 30, 4187-4192.

9. Yu, C.H.; Kan, S.F.; Pu, H.F.; Jea Chien, E.; Wang, P.S. Apoptotic signaling in bufalin- and cinobufagin-treated androgen-dependent and -independent human prostate cancer cells. Cancer Sci. 2008, 99, 2467-2476.

10. Takai, N.; Kira, N.; Ishii, T.; Yoshida, T.; Nishida, M.; Nishida, Y.; Nasu, K.; Narahara, H. Bufalin, a traditional oriental medicine, induces apoptosis in human cancer cells. Asian Pac. J. Cancer Prev. 2012, 13, 399-402.

11. Huang, H.; Cao, Y.; Wei, W.; Liu, W.; Lu, S.Y.; Chen, Y.B.; Wang, Y.; Yan, H.; Wu, Y.L. Targeting poly (ADP-ribose) polymerase partially contributes to bufalin-induced cell death in multiple myeloma cells. PLoS One 2013, 8, e66130.

12. Nasu, K.; Nishida, M.; Ueda, T.; Takai, N.; Bing, S.; Narahara, H.; Miyakawa, I. Bufalin induces apoptosis and the G0/G1 cell cycle arrest of endometriotic stromal cells: A promising agent for the treatment of endometriosis. Mol. Hum. Reprod. 2005, 11, 817-823.

13. Cao, H.; Shibayama-Imazu, T.; Masuda, Y.; Shinki, T.; Nakajo, S.; Nakaya, K. Involvement of Tiam1 in apoptosis induced by bufalin in HeLa cells. Anticancer Res. 2007, 27, 245-249.

14. Masuda, Y.; Kawazoe, N.; Nakajo, S.; Yoshida, T.; Kuroiwa, Y.; Nakaya, K. Bufalin induces apoptosis and influences the expression of apoptosis-related genes in human leukemia cells. Leuk. Res. 1995, 19, 549-556.

15. Lee, J.H.; Koh, Y.A.; Cho, C.K.; Lee, S.J.; Lee, Y.S.; Bae, S. Identification of a novel ionizing radiation-induced nuclease, AEN, and its functional characterization in apoptosis. Biochem. Biophys. Res. Commun. 2005, 337, 39-47.

16. Kawase, T.; Ichikawa, H.; Ohta, T.; Nozaki, N.; Tashiro, F.; Ohki, R.; Taya, Y. p53 target gene AEN is a nuclear exonuclease required for p53-dependent apoptosis. Oncogene 2008, 27, 3797-3810.

17. Zumbrun, S.D.; Hoffman, B.; Liebermann, D.A. Distinct mechanisms are utilized to induce stress sensor gadd45b by different stress stimuli. J. Cell. Biochem. 2009, 108, 1220-1231. 
18. Vairapandi, M.; Balliet, A.G.; Hoffman, B.; Liebermann, D.A. GADD45b and GADD45g are cdc2/cyclinB1 kinase inhibitors with a role in $\mathrm{S}$ and $\mathrm{G} 2 / \mathrm{M}$ cell cycle checkpoints induced by genotoxic stress. J. Cell. Physiol. 2002, 192, 327-38.

19. Zenmyo, M.; Tanimoto, A.; Sakakima, H.; Yokouchi, M.; Nagano, S.; Yamamoto, T.; Ishido, Y.; Komiya, S.; Ijiri, K. Gadd45beta expression in chondrosarcoma: A pilot study for diagnostic and biological implications in histological grading. Diagn. Pathol. 2010, 5, 69-73.

20. Richie, C.T.; Peterson, C.; Lu, T.; Hittelman, W.N.; Carpenter, P.B.; Legerski, R.J. hSnm1 colocalizes and physically associates with 53BP1 before and after DNA damage. Mol. Cell. Biol. 2002, 22, 8635-8647.

21. Akhter, S.; Richie, C.T.; Deng, J.M.; Brey, E.; Zhang, X.; Patrick, C., Jr.; Behringer, R.R.; Legerski, R.J. Deficiency in SNM1 abolishes an early mitotic checkpoint induced by spindle stress. Mol. Cell. Biol. 2004, 24, 10448-10455.

22. Koyama-Nasu, R.; Nasu-Nishimura, Y.; Todo, T.; Ino, Y.; Saito, N.; Aburatani, H.; Funato, K.; Echizen, K.; Sugano, H.; Haruta, R.; et al. The critical role of cyclin D2 in cell cycle progression and tumorigenicity of glioblastoma stem cells. Oncogene 2013, 32, 3840-3845.

23. Igawa, T.; Sato, Y.; Takata, K.; Fushimi, S.; Tamura, M.; Nakamura, N.; Maeda, Y.; Orita, Y.; Tanimoto, M.; Yoshino, T. Cyclin D2 is overexpressed in proliferation centers of chronic lymphocytic leukemia/small lymphocytic lymphoma. Cancer Sci. 2011, 102, 2103-2107.

24. Liu, S.C.; Bassi, D.E.; Zhang, S.Y.; Holoran, D.; Conti, C.J.; Klein-Szanto, A.J. Overexpression of cyclin D2 is associated with increased in vivo invasiveness of human squamous carcinoma cells. Mol. Carcinog. 2002, 34, 131-139.

25. Chang, Y.-M.; Velmurugan, B.K.; Kuo, W.-W.; Chen, Y.-S.; Ho, T.-J.; Tsai, C.-T.; Ye, C.-X.; Tsai, C.-H.; Tsai, F.-J.; Huang, C.-Y. Inhibitory effect of alpinate Oxyphyllae fructus extracts on Ang II-induced cardiac pathological remodeling-related pathways in $\mathrm{H} 9 \mathrm{c} 2$ cardiomyoblast cells. Biomedicine 2013, 3, 148-152.

26. Lin, M.-C.; Tsai, S.-Y.; Wang, F.-Y.; Liu, F.-H.; Syu, J.-N.; Tang, F.-Y. Leptin induces cell invasion and the upregulation of matrilysin in human colon cancer cells. Biomedicine 2013, 3, 174-180.

27. Leung, Y.M.; Wong, K.L.; Chen, S.W.; Lu, D.Y.; Kuo, C.S.; Chen, Y.R.; Chen, Y.W.; Cheng, T.H. Down-regulation of voltage-gated $\mathrm{Ca} 2+$ channels in $\mathrm{Ca} 2+$ store-depleted rat insulinoma RINm5F cells. Biomedicine 2013, 3, 130-139.

28. Wu, R.S.; Liu, K.C.; Tang, N.Y.; Chung, H.K.; Ip, S.W.; Yang, J.S.; Chung, J.G. cDNA microarray analysis of the gene expression of murine leukemia RAW 264.7 cells after exposure to propofol. Environ. Toxicol. 2013, 28, 471-478.

29. Gardina, P.J.; Clark, T.A.; Shimada, B.; Staples, M.K.; Yang, Q.; Veitch, J.; Schweitzer, A.; Awad, T.; Sugnet, C.; Dee, S.; et al. Alternative splicing and differential gene expression in colon cancer detected by a whole genome exon array. BMC Genomics 2006, 7, 325.

30. Douglas, D.; Hsu, J.H.; Hung, L.; Cooper, A.; Abdueva, D.; van Doorninck, J.; Peng, G.; Shimada, H.; Triche, T.J.; Lawlor, E.R. BMI-1 promotes ewing sarcoma tumorigenicity independent of CDKN2A repression. Cancer Res. 2008, 68, 6507-6515. 
31. Jacobs, A.T.; Marnett, L.J. HSF1-mediated BAG3 expression attenuates apoptosis in 4-hydroxynonenal-treated colon cancer cells via stabilization of anti-apoptotic Bcl-2 proteins. J. Biol. Chem. 2009, 284, 9176-9183.

Sample Availability: Not available.

(C) 2014 by the authors; licensee MDPI, Basel, Switzerland. This article is an open access article distributed under the terms and conditions of the Creative Commons Attribution license (http://creativecommons.org/licenses/by/3.0/). 